\title{
Some more equal than others
}

\section{Publishers have varying policies as to when published articles become freely available to the public, but it seems that within any one publishing group the free access policy should be the same for all authors.}

$\mathbf{S}$ ince the launch of PubMed Central, the US National Institutes of Health (NIH) free digital archive of biomedical research, in 2000, the participation of publishers has been voluntary. However, in response to the demands of an increasing number of funding agencies to make their grantees' papers freely available online, most publishers have had to adopt some kind of open access policy. Some journals make their content available in PubMed Central immediately upon publication, whereas others delay release for anywhere from a few months to a few years after publication. However, within any one journal, the policies are the same for all authors.

Over the past few years, though, a multitiered system has emerged at Cell Press, in which who funds your work directly affects how soon your work becomes publicly available and how much open access will cost.

The most recent deal is between the Howard Hughes Medical Institute (HHMI) and Elsevier. Starting 1 September 2007, HHMI will pay Elsevier \$1,000 per Cell Press article and \$1,500 per Elsevier article to deposit the author's manuscript in PubMed Central six months after publication. The author's manuscript will have been through the peer review process and accepted for publication but will not have undergone editing and formatting. The agreement was prompted by a policy that HHMI is about to adopt, under which all HHMI-funded research must be made available in an open access repository six months after publication.

On the other hand, if you happen to be funded by the Wellcome Trust, your paper will be made freely available upon publication in Cell Press, for a price. The Wellcome Trust requires that authors' work be made freely available to the public, through PubMed Central, no later than six months after the official date of final publication. In the deal struck with Elsevier last fall, the Wellcome Trust is getting immediate public access to the published version of the paper (after peer-review and copyediting) through PubMed Central, but it is paying $\$ 3,000$ per Elsevier article and \$5,000 per Cell Press article.

Meanwhile, Cell Press authors who are NIH funded fall under a third access policy and have the option of having authors' versions of their papers (peer reviewed but not copyedited) posted to PubMed Central 12 months after publication; but given that Cell Press papers are freely available after one year on Cell's website, this seems somewhat beside the point.

If you are not funded by any of the above three agencies and you publish a Cell Press article, you can post a revised personal version of the final text of the article (to reflect changes made in the peer-review and editing process) on your personal or institutional website or server, with a link to the article as published, but not in PubMed Central.

By comparison, all journals published by the Nature Publishing Group, including the Nature journals, encourage deposition of the authors' versions of accepted papers (the unedited manuscripts) in PubMed Central, in institutional repositories and on authors' personal websites, six months after publication. Deposition of an article should include a link to the published article on the journal's website.

Science's access policy states, "After publication, authors may post the accepted version of the paper on the author's personal Web site and are provided one referrer link that can be posted on a personal or institutional Web page, through which users can freely access the published paper on Science's Web site. Science allows deposition of accepted papers in PubMed Central 6 months after publication, in accord with the requirements of the funders NIH and the Wellcome Trust, provided that a link to the final version published in Science is included." After one year, Science opens its articles to free access on its website.

Of course, there have been many lively and sometimes heated debates about when papers should be made publicly available after publication $(0,6$ or 12 months), where (in PubMed Central or on the journal's website) and in what form (the author's version or the published version). There are also commercial and not-for-profit publishers that produce peer-reviewed journals that follow different business models. For example, there are 'subscriber-pays' models in which journals are paid for by readers, libraries and similar institutions, and 'author-pays' models in which publication is paid for by the author, or the author's institution or funder. There are also open access journals where everything published is freely available online, and others where the research articles are open access but subscriptions are required for other content — and then there are journals where the content is subscription based but authors can pay for immediate open access to the published versions of their papers. There are variations on all of these themes, but within any one journal or publishing group, the same options are available to all authors regardless of the source of their funding.

There are inequalities in science (as in life) that are difficult to avoid, but this should not be one of them. A system of public access to published work should provide a level playing field to all authors who publish in a given journal. Not doing so introduces an unnecessarily tiered system that does not best serve the interests of authors or readers. 